\title{
Sonication contribution to identifying prosthetic joint infection with Ralstonia pickettii: a case report and review of the literature
}

Rares Mircea Birlutiu*, Mihai Dan Roman², Razvan Silviu Cismasiu³ ${ }^{3}$ Sorin Radu Fleaca², Crina Maria Popa ${ }^{4}$, Manuela Mihalache ${ }^{5}$ and Victoria Birlutiu ${ }^{6}$

\begin{abstract}
Background: In the context of an increase number of primary and revision total hip and total knee arthroplasty performed yearly, an increased risk of complication is expected. Prosthetic joint infection (PJI) remains the most common and feared arthroplasty complication. Ralstonia pickettii is a Gram-negative bacterium, that has also been identified in biofilms. It remains an extremely rare cause of PJI. There is no report of an identification of $R$. pickettii on an extracted spacer loaded with antibiotic.

Case presentation: We present the case of an 83-years-old Caucasian male patient, that underwent a right cemented total hip replacement surgery. The patient is diagnosed with an early PJI with no isolated microorganism. A debridement and change of mobile parts is performed. At the beginning of 2016, the patient in readmitted into the Orthopedic Department for sever, right abdominal and groin pain and elevated serum erythrocyte sedimentation rate and C-reactive protein. A joint aspiration is performed with a negative microbiological examination. A two-stage exchange with long interval management is adopted, and a preformed spacer loaded with gentamicin was implanted. In July 2016, based on the proinflammatory markers evolution, a shift a three-stage exchange strategy is decided. In September 2016, a debridement, and changing of the preformed spacer loaded with gentamicin with another was carried out. Bacteriological examination of the tissues sampled intraoperatively was positive for Pseudomonas aeruginosa. From the sonication fluid, no bacteria were isolated on culture or identified using the bbFISH assay. During the hospitalization period, the patient received i.v. ceftazidime $3 \times 2 \mathrm{~g} /$ day and p.o. ciprofloxacin $2 \times 750 \mathrm{mg} /$ day, antibiotic therapy that was continued after discharge with p.o. ciprofloxacin 2x750mg/day for 6 weeks. In February 2017, a reimplantation of a revision prosthesis is performed. The retrieved spacer is sonicated, and after 4 days of incubation of the sonication fluid, R. pickettii is isolated. A long term antibiotic therapy with cotrimoxazole being prescribed.
\end{abstract}

Conclusions: Bacteria culture of sonication fluid remains the gold standard in diagnosing prosthetic joint infections. R. pickettii remains an extremely rare cause of prosthetic joint infection. Optimal management of $R$. pickettii prosthetic joint infections of has not been established.

Keywords: Ralstonia pickettii, Prosthetic joint infection, Sonication, Biofilm, Spacer, Case report

\footnotetext{
* Correspondence: raresmircea@gmail.com

${ }^{1}$ Lucian Blaga University of Sibiu, Faculty of Medicine Sibiu; FOISOR Clinical

Hospital of Orthopedics, Traumatology, and Osteoarticular TB Bucharest,

Address: Str. Lucian Blaga, Nr. 2A, 550169 Sibiu, Romania

Full list of author information is available at the end of the article
} 


\section{Background}

Orthopedic surgery of total hip and total knee arthroplasty were the most successful orthopedic surgeries of the last century. In the context of an increase number of primary and revision total hip and total knee arthroplasty performed each year, there it is expected an increased risk of complication. Prosthetic joint infection (PJI) remains the most common and feared arthroplasty complication. A correct diagnosis of infection is decisive for a correct treatment of orthopedic implant-related infections.

The diagnosis of PJI is based on history of the patient (fever, pain, surgeries in the past), clinical assessment (the presence of a sinus tract) and laboratory tests. The laboratory criteria of PJI diagnosis have not yet been established.

Ralstonia pickettii, is a Gram-negative bacilli, rodshaped, non-fermentive, soli bacterium, that can cause severe nosocomial infections, in immunocompromised patients (premature infants, Crohn's disease, cystic fibrosis, leukemia), like meningitis, sepsis, pneumonia, and osteoarticular infection through contamination of the sterile water, saline solution, disinfectants, etc. Prosthetic joint infection caused by $R$. pickettii is rarely reported in literature, a few cases have been described, there is no report of an identification of $R$. pickettii on an extracted spacer loaded with antibiotic (gentamicin).

\section{Case presentation}

We present the case of an 83-years-old Caucasian male patient, known with high blood pressure, mitral valve regurgitation, coronary artery disease, diabetes mellitus type 2, that underwent a right cemented total hip replacement surgery for osteoarthritis in 2014 in the Academic Emergency Hospital Sibiu, Romania. Detailed clinical patient information is given in Table 1 . Three weeks after the surgery, the patient is diagnosed with an early PJI with no isolated microorganism from periprosthetic tissues or fluids harvested during the surgery. A debridement and change of mobile parts with the retention of fixed prosthetic components is performed. Soft tissues surrounding the implant and periprosthetic interface membrane were taken for histopathological testing. The patient was discharged from the hospital and treated as an outpatient with cefuroxime $2 \times 500 \mathrm{mg} /$ day for 2 weeks.

At the beginning of 2016, the patient is readmitted into the Orthopedic Department for sever, right abdominal and groin pain, and elevated serum erythrocyte sedimentation rate and C-reactive protein. An anteriorposterior (AP) radiograph of the pelvis and a horizontal beam lateral hip radiograph of the right hip are performed, reveling signs of loosening at the level of both the acetabular and femoral components. A joint aspiration is performed with a negative microbiological examination. A two-stage exchange with long interval management is adopted, and a preformed spacer loaded with gentamicin was implanted. Intraoperative tissue samples are harvested and Pseudomonas aeruginosa is isolated. Oral ciprofloxacin $2 \times 750 \mathrm{mg} /$ day is administered for 6 weeks and intravenous (i.v.) gentamicin $1 \times 240 \mathrm{mg} /$ day for 2 weeks after the discharge from the hospital, according to the results of the susceptibility tests that were performed.

The patient was readmitted in one of the Internal Medicine Department, after 3 months, for right groin pain and altered proinflammatory markers, for which nonsteroidal anti-inflammatory drugs, muscle relaxants, and anti-clotting drugs are administered with favorable outcome.

In July 2016, based on the proinflammatory markers evolution, a shift a three-stage exchange strategy is decided. A joint aspiration is performed with a negative microbiological examination. A debridement, and changing of the preformed spacer loaded with gentamicin with another was carried out. Intraoperatively soft tissues were taken for histopathological testing. Oral ciprofloxacin $2 \times 500 \mathrm{mg} /$ day is administered for 3 weeks, according to the results of the susceptibility tests that were performed.

With the beginning of September 2016, a new strategy for the diagnostics and management of prosthetic joint infection is implemented in the Academic Emergency Hospital Sibiu. Strategy that includes the sonication of the retrieved implant (prosthesis or polymethylmethacrylate spacer). In the operating theater, Ringer's or saline sterile solution in added in the sterile containers. The implants are processed within $30 \mathrm{~min}$ by sonication (1 min) using an ultrasound bath (BactoSonic ${ }^{\circledR} 14.2$, Bandelin $\mathrm{GmbH}$, Berlin, Germany) at a frequency of $42 \mathrm{kHz}$ and a power density of $0.22 \mathrm{~W} / \mathrm{cm}^{2}$. The resulting sonication fluid is vortexed, and $50 \mathrm{ml}$ of sonication fluid is centrifuged at $2500 \mathrm{rpm}$ for $5 \mathrm{~min}$. The resulted precipitate is inoculated onto Columbia agar with sheep blood (incubated aerobically, anaerobically and in high concentration of $\mathrm{CO}_{2}$ ), Sabouraud plate, MacConkey agar plate, glucose broth, lactose broth and thioglycollate broth. Cultures are incubated at $37^{\circ} \mathrm{C}$ for 14 days and inspected daily for bacterial growth. Isolated bacteria are identified using the VITEK 2 Compact analyzer (bioMérieux, Marcy-l'Étoile, France). The MICs (minimum inhibitory concentrations) are assessed according to the European Committee on Antimicrobial Susceptibility Testing breakpoints. In addition, as a rapid method of bacteria detection, molecular identification of bacteria by $16 \mathrm{~S}$ rRNA bbFISH (beacon-based fluorescent in situ hybridization) technology using a bbFISH kit (hemoFISH ${ }^{\ominus}$ Masterpanel, miacom diagnostics $\mathrm{GmbH}$ Düsseldorf, Germany), was 


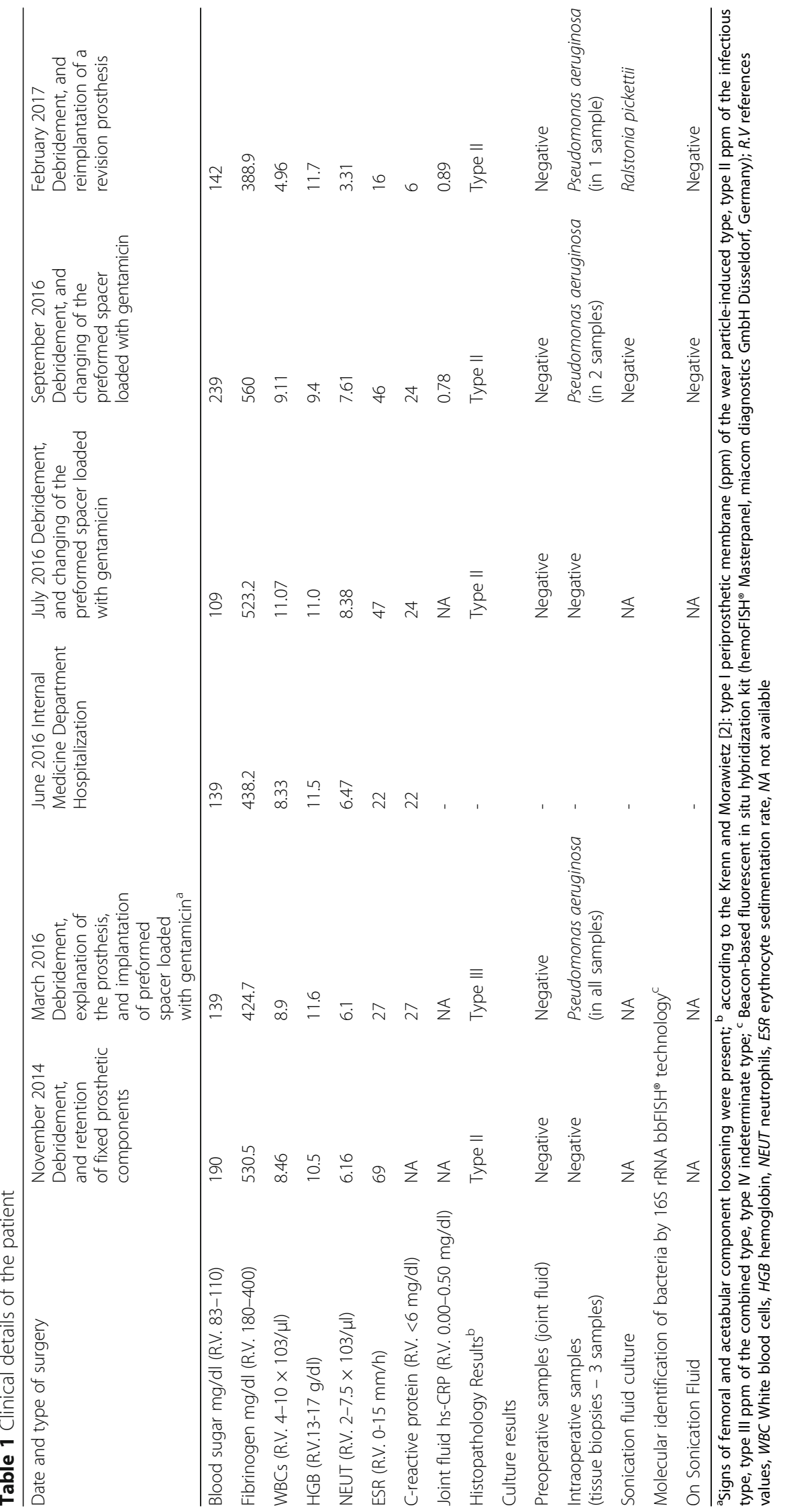


also implemented. The FISH assay are performed according to the manufacturer using a sample of resulted precipitate from $50 \mathrm{ml}$ of sonication fluid that is centrifuged at $2500 \mathrm{rpm}$ for $5 \mathrm{~min}$. The kit contains beacons for the detection of Staphylococcus spp., Staphylococcus aureus, Streptococcus spp., Streptococcus pneumoniae, Streptococcus agalactiae, Enterococcus faecium, Enterococcus faecalis, Enterobacteriaceae, Escherichia coli, Klebsiella pneumoniae, Proteus mirabilis, Pseudomonas aeruginosa, Acinetobacter spp., and Stenotrophomonas maltophilia.

In September 2016, a joint aspiration is performed with a negative microbiological examination. A debridement, and changing of the preformed spacer loaded with gentamicin with another was carried out. Soft tissues surrounding the implant and periprosthetic interface membrane were taken intraoperatively for histopathological testing. Bacteriological examination of the tissues sampled intraoperatively was positive for Pseudomonas aeruginosa, strain that was identified using the VITEK 2 Compact analyzer (bioMérieux, Marcy-l'Étoile, France) and was sensitive to amikacin, cefepime, ceftazidime, ciprofloxacin, pefloxacin, Piperacillin, ticarcillin, meropenem, imipenem, colistin, gentamicin resistant to trimethoprim/ sulfamethoxazole. From the sonication fluid, no bacteria were isolated on culture or identified using the bbFISH assay. During the hospitalization period, the patient received i.v. ceftazidim $3 \times 2$ g/day and p.o. ciprofloxacin $2 \times 750 \mathrm{mg} /$ day, antibiotic therapy that was continued at home after discharge with p.o. ciprofloxacin $2 \times 750 \mathrm{mg} /$ day for 6 weeks.

After 5 months, in February 2017, based on the serum proinflammatory markers levels and after a joint aspiration was performed with a negative microbiological examination, a debridement, and reimplantation of a revision prosthesis is performed. Soft tissues surrounding the implant and periprosthetic interface membrane were taken intraoperatively for histopathological testing. Bacteriological examination of the tissues sampled intraoperatively was positive for Pseudomonas aeruginosa, strain with the same susceptibility as the one isolated earlier. The retrieved spacer is sonicated. From the sonication fluid, after 4 days form the inoculation of the sonication fluid on the growth medium, Ralstonia pickettii was isolated. The isolated strain of $R$. pickettii was sensitive to ticarcillin, piperacillin, cefepime, imipenem, meropenem, ciprofloxacin, pefloxacin, minocycline, cotrimoxazole, resistant to aztreonam, amikacin, gentamicin, colistin, and intermediate sensitive to ceftazidime. No bacteria were identified using the bbFISH assay. The antibiotic therapy with p.o. cotrimoxazole $3 \times 2$ tb $(80 \mathrm{mg}$ trimethoprim bp and $400 \mathrm{mg}$ sulfamethoxazole bp)/day is initiated during the hospitalization period, and will be administered for a total duration of 12 weeks, the patient is discharged after 10 days from the admission into the hospital and is scheduled for routine follow-ups. On discharge, the wound is healed, with no open areas, and with sutures that are intact (and will be removed at 21 days after the surgery). The wound edges are wellapproximated. The skin surrounding the wound is viable and clean. The wound looks good with no drainage. The dressing was clean and dry. The patient evolution, undertreatment, is favorable. At the last follow-up visit at (14 weeks after the surgery) the wound is healed, with no open areas, and with a clean skin surrounding the wound. The patient is using a cane for long walks, and with no clinical complaints. The laboratory investigations (white blood cells count, fibrinogen, C-reactive protein, erythrocyte sedimentation rate, creatinine/estimated glomerular filtration rate (eGFR), liver enzymes (aspartate aminotransferase - AST/SGOT and alanine aminotransferase - ALT/SGPT)) were performed on regular base and were within references levels.

\section{Discussions}

Ralstonia pickettii, also known previously under other names like Burkholderia pickettii, B. solanacearum, Alcaligenes eutrophupickettii is part of Betaproteobacteria class, Burkholderiales order, Ralstoiaceae family, Ralstonia genus, is a Gram-negative, nonfermentative, oxidase-positive bacteria. The genus Ralstonia includes 10 other bacteria: $R$. insidiosa [1], R. solanacearum, $R$. mannitolilytica [2], $R$. eutropha, $R$. gilardii, $R$. paucula, $R$. basilensis, $R$. oxalate, $R$. taiwanensis, $R$. campinensis, and $R$. metallidurans [3-7]. It is an opportunistic pathogen associated with nosocomial infections due to contamination of sterile water, saline solution [8,9], disinfectants, blood culture bottles, and venous catheters. Exceptionally being isolated from the mouth and upper respiratory tract [4], being responsible for lung abscess after necrotizing pneumonia in elderly, non-hospitalized patients [10] or lobar pneumonia associated with severe respiratory insufficiency [11].

R.pickettii is present in the environment, in water, plants, in enriched in heavy metals soil; may be associated in biofilm, being able to grow in nutrient deficient conditions. It can affect immunocompromised hosts, especially patients with Crohn's disease or cystic fibrosis [12]. So far, around 70 cases of infection caused by $R$. pickettii, in humans, have been published in the literature. There are described cases of systemic infections in premature infants like bacteremia [13], infection associated with the administration contaminated heparin of flush [14], infection that tend to evolve, potentially severe, in infants, with severe sepsis, multiple organ failure, and disseminated intravascular coagulation [15]. R. pickettii bacteremia, was associated also with the use of contaminated antiseptic solutions - chlorhexidine solutions, and 
distilled water or with extracorporeal membrane oxygenation [16], and with the contamination of irrigation system in obstetric care [17]. $R$. pickettii was identified also in septic arthritis, spinal osteitis [18], osteomyelitis [19], meningitis [20] or ventricularperitoneal shunt infection [21]. In adults, fatal cases have been described in hematological patients (e.g. patients with leukemia) with immunosuppressive therapy and in patients with thalassemia that develop infection [22]. R. pickettii infection was also identified in patients with cochlear implants [23]. Identification in the male genital area appears to be associated with secondary infertility [24]. According to the data published in the literature, orthopedic implant-associated infection rate is between 1 and $4 \%$ for primary total joint arthroplasty $[25,26]$, nearly doubling $(7 \%)$ for revision surgeries [25]. There are few cases of PJIs in which $R$. pickettii was isolated: Gomez-Barrena E et al. [27] identified 3 cases of infections associated with total knee arthroplasty, Lepetsos et al. published a case knee PJI [28], and Przemysław L [29], confirms the involvement in 3 cases of PJI, two knees and one hip PJIs. Esteban J et al. [30] identifies infections caused by $R$. pickettii after using sonication on intramedullary nails. A similar case like ours, in which $R$. pickettii to associated with an antibiotic spacer, was not published so far in the literature. According to other studies [31], identification of $R$. pickettii after sonication of the implant was superior to joint fluid cultures. In our case, the lack of identifying the strain in the previous step of the revision surgery, in which sonication was also used, suggests a potential recent infection, possibly being associated with the saline lavage during the debridement stage of the surgery. The antibiotic sensitivity, of the isolated strain, was at quinolones and trimethoprim/sulfamethoxazole, antibiotics used to treat the patient. The resistance of the strains to gentamicin, that was found in the spacers loaded with antibiotics, explains the growth of $R$. pickettii at this level. It is necessary to better assess in terms of bacteriological examination of the prosthesis, by sonication and molecular detection, and in terms of the local treatment options, options that should be efficient also against opportunistic pathogens resistant to aminoglycosides, or vancomycin, antibiotics that are commonly used in revision surgeries.

\section{Conclusions}

In conclusion, bacteria culture of sonication fluid remains the gold standard in diagnosing prosthetic joint infections. Negative culture of preoperative joint aspiration and soft tissues surrounding the implant and periprosthetic interface membrane obtained intraoperatively, do not exclude the presence of bacteria on the implants. Ralstonia pickettii remains an extremely rare cause of prosthetic joint infection. The introduction of ciprofloxacin in spacers in association with aminoglycosides or vancomycin, seems to be a better option to extend the antibacterial effectiveness against opportunistic germs.

\section{Abbreviations}

ALT/SGPT: alanine aminotransferase; AP: Anteriorposterior; AST/

SGOT: aspartate aminotransferase; bbFISH: Beacon-based fluorescent in situ hybridization; eGFR: creatinine/estimated glomerular filtration rate;

i.v: Intravenous; MICs: Minimum inhibitory concentrations; PJI: Prosthetic joint infection

\section{Acknowledgements}

We express our special thanks to the medical staff from the Infectious Disease Department, and the Orthopedics and Traumatology Department of the Academic Emergency Hospital from Sibiu, and to the staff from Polisano European Hospital Sibiu, Romania.

\section{Funding}

The authors received no funding for the manuscript preparation or for publishing the article.

\section{Availability of data and materials}

The authors do not wish to share their data, they respect the patient rights to privacy and to protect his identity. The authors presented, in the manuscript, all the necessary information about their case report. Raw data regarding our patient is in his admission file, file that is strictly confidential, without the possibility of publishing raw data from it.

\section{Authors' contributions}

All authors (RMB, MDR, RSC, SRF, CMP, MM, and VB) made contribution in equal parts to this manuscript in terms of acquisition, analysis and interpretation of data, conception and design, and drafting the manuscript. All authors were involved in the therapeutic management of the patient. All authors were involved in revising the manuscript. All authors read and approved the final version of the manuscript.

\section{Ethics approval and consent to participate}

Written informed consent was obtained from the patient for publication of this case report and any accompanying images. The study was accepted by the Ethics Committee of the hospital and they encouraged publishing the article. A copy of the written consent is available for review by the Editor-inChief of this journal.

\section{Competing interests}

The authors declare that they have no competing interests.

\section{Publisher's Note}

Springer Nature remains neutral with regard to jurisdictional claims in published maps and institutional affiliations.

\section{Author details}

${ }^{1}$ Lucian Blaga University of Sibiu, Faculty of Medicine Sibiu; FOISOR Clinical Hospital of Orthopedics, Traumatology, and Osteoarticular TB Bucharest, Address: Str. Lucian Blaga, Nr. 2A, 550169 Sibiu, Romania. 'Lucian Blaga University of Sibiu, Faculty of Medicine Sibiu, Academic Emergency Hospital Sibiu - Orthopedics and Traumatology Department, Address: Str. Lucian Blaga, Nr. 2A, 550169 Sibiu, Romania. ${ }^{3}$ Carol Davila University of Medicine and Pharmacy Bucharest, Romania; FOISOR Clinical Hospital of Orthopedics, Traumatology, and Osteoarticular TB Bucharest, Address: Str. Dionisie Lupu nr. 37, 020021 Bucharest, Sector 2, Romania. ${ }^{4}$ Polisano European Hospital Sibiu, Address: Str. Izvorului Nr. 1A, Sibiu, Romania. ${ }^{5}$ Lucian Blaga University of Sibiu, Faculty of Medicine Sibiu, Address: Str. Lucian Blaga, Nr. 2A, 550169 Sibiu, Romania. ${ }^{6}$ Lucian Blaga University of Sibiu, Faculty of Medicine Sibiu, Academic Emergency Hospital Sibiu - Chief of the Infectious Diseases Departmen, Address: Str. Lucian Blaga, Nr. 2A, 550169 Sibiu, Romania. 
Received: 27 March 2017 Accepted: 13 July 2017

Published online: 19 July 2017

\section{References}

1. Morawietz L, Classen RA, Schröder JH, Dynybil C, Perka C, Skwara A, et al. Proposal for a histopathological consensus classification of the periprosthetic interface membrane. J Clin Pathol. 2006;59(6):591-7.

2. Ryan MP, Pembroke JT, Adley CC. Differentiating the growing nosocomial infectious threats Ralstonia pickettii and Ralstonia insidiosa. Eur J Clin Microbiol Infect Dis. 2011;30(10):1245-7. doi:10.1007/s10096-011-1219-9.

3. Daxboeck F, Stadler M, Assadian O, Marko E, Hirschl AM, Koller W. Characterization of clinically isolated Ralstonia mannitolilytica strains using random amplification of polymorphic DNA (RAPD) typing and antimicrobial sensitivity, and comparison of the classification efficacy of phenotypic and genotypic assays. J Med Microbiol. 2005;54(Pt 1):55-61. doi:10.1099/jmm.0.45656-0.

4. Coenye T, Vandamme P, LiPuma J. Infection by Ralstonia species in cystic fibrosis patients: identification of R. pickettii and R. mannitolilytica by polymerase chain reaction. Emerg Infect Dis. 2002;8(7):692-6.

5. Sahin N, Isik K, Tamer AU, Goodfellow M. Taxonomic position of "pseudomonas oxalaticus" strain ox14T (DSM 1105T) (Khambata and Bhat, 1953) and its description in the genus Ralstonia as Ralstonia oxalatica comb. nov. Syst Appl Microbiol. 2000;23(2):206-9.

6. De Baere T, Steyaert S, Wauters G, De Vos P, Goris J, Coenye T, et al. Classification of Ralstonia picketii biovar 3/'thomasii' strains (Pickett 1994) and of new isolates related to nosocomial recurrent meningitis as Ralstonia mannitolytica sp. nov. Int J Syst Evol Microbiol. 2001:51:547-58.

7. Chen WM, Laevens S, Lee TM, Coenye T, De Vos P, Mergeay M, et al. Ralstonia taiwanensis sp. nov., isolated from root nodules of mimosa species and sputum of a cystic fibrosis patient. Int J Syst Evol Microbiol. 2001; 51(Pt2):1729-35.

8. Goris J, De Vos P, Coenye T, Hoste B, Janssens D, Brim H, et al. Classification of metal-resistant bacteria from industrial biotopes as Ralstonia campinensis sp. nov., Ralstonia metallidurans sp. nov. and Ralstonia basilensis Steinle et al. 1998 emend. Int J Syst Evol Microbiol. 2001;51(Pt5):1773-82.

9. Labarca JA, Trick WE, Peterson CL, Carson LA, Holt SC, Arduino MJ, et al. A multistate nosocomial outbreak of Ralstonia pickettii colonization associated with an intrinsically contaminated respiratory care solution. Clin Infect Dis. 1999;29(5):1281-6.

10. Centers for Disease Control and Prevention (CDC). Nosocomial Ralstonia pickettii colonization associated with intrinsically contaminated saline solution_Los Angeles, California, 1998. MMWR Morb Mortal Wkly Rep. 1998;47(14):285-6.

11. Pan W, Zhao Z, Dong M. Lobar pneumonia caused by Ralstonia pickettii in a sixty-five-year-old Han Chinese man: a case report. J Med Case Reports. 2011;5:377. doi:10.1186/1752-1947-5-377.

12. Küçükbayrak A, Uğurman F, Dereli N, Cizmeci Z, Günay E. A community acquired pneumonia case caused by Ralstonia pickettii. Mikrobiyol Bul. 2009; 43(2):331-4.

13. Coenye T, De Vos P, Goris J, Vandamme P, LiPuma JJ. Classification of Ralstonia pickettii-like isolates from the environment and clinical samples as Ralstonia insidiosa. Int J Syst Evol Microbiol. 2003;53(Pt 4):1075-80.

14. Sharma D, Sharma P, Soni P, Gupta B. Ralstonia picketti neonatal sepsis: a case report. BMC Res Notes. 2017;10(1):28. doi:10.1186/s13104-016-2347-1.

15. Kimura AC, Calvet H, Higa J, Pitt H, Frank C, Padilla G, et al. Outbreak of Ralstonia pickettii bacteremia in a neonatal intensive care unit. Pediatr Infect Dis J. 2005;24(12):1099-103.

16. Vitaliti SM, Maggio MC, Cipolla D, Corsello G, Mammina C. Neonatal sepsis caused by Ralstonia pickettii. Pediatr Infect Dis J. 2008;27(3):283. doi:10.1097/ INF.0b013e31816454b7.

17. Riley PS, Weaver RE. Recognition of pseudomonas pickettii in the clinical laboratory: biochemical characterization of 62 strains. J Clin Microbiol. 1975;1(1):61-4.

18. Yoneyama A, Yano H, Hitomi S, Okuzumi K, Suzuki R, Kimura S. Ralstonia pickettii colonization of patients in an obstetric ward caused by a contaminated irrigation system. J Hosp Infect. 2000;46:79-80.

19. Elsner HA, Dahmen GP, Laufs R, Mack D. Ralstonia pickettii involved in spinal osteitis in an immunocompetent adult. J Inf Secur. 1998;36(3):352.

20. Degeorges R, Teboul F, Belkheyar Z, Oberlin C. Ralstonia pickettii osteomyelitis of the trapezium. Chir Main. 2005;24:174-6.

21. Heagney MA. An unusual case of bacterial meningitis caused by Burkholderia pickettii. Chir Main. 2005;24(3-4):174-6.
22. Bonatti H, Stelzmueller I, Laimer I, Obwegeser A. Ralstonia pickettii meningitis in a child with hydrocephalus. Eur J Pediatr Surg. 2009:19(5):3412. doi:10.1055/s-0029-1202252.

23. Pandey R, Barman P, Sengupta S. Ralstonia Pickettii Bacteremia. J Infect Dis Ther. 2014;2:179. http://dx.doi.org/10.4172/2332-0877.1000179

24. Varadarajan W, Dirain CO, Antonelli PJ. Microflora of retained Intracochlear electrodes from infected Cochlear implants. Otolaryngol Head Neck Surg. 2017; doi:10.1177/0194599817693228. [Epub ahead of print]

25. Carrell DT, Emery BR, Hamilton B. Seminal infection with Ralstonia pickettii and cytolysosomal spermophagy in a previously fertile man. Fertil Steril. 2003;79:1665-7.

26. AAOS Guideline on the Diagnosis of Periprosthetic Joint Infection of the Hip and Knee, Guideline and Evidence Report adopted by American Academy of Orthopaedic Surgeons, Board of Directors, 2010. Available via http://www.aaos.org/. Accessed 24 March 2017.

27. Parvizi J, Walinchus L, Adeli B. Molecular diagnostics in periprosthetic joint infection. Int J Artif Organs. 2011;34(9):847-55.

28. Gomez-Barrena E, Esteban J, Medel F, Molina-Manso D, Ortiz-Perez A, Jose Cordero-Ampuero JA. Bacterial adherence to separated modular components in joint prosthesis: a clinical study. J Orthop Res. 2012;30:1634-9.

29. Lepetsos P, Stylianakis A, Michail S, Argyris D, Lelekis M, Anastasopoulos P. Periprostheti knee infection caused by Ralstonia pickettii leading to knee arthrodesis. A case report and review of the literature. P28 - (\#220) - poster. 34th Annual Meeting of the European Bone\&Joint Infection Society, Estoril Lisboa Portugal, Sept 10-12, 2015.

30. Esteban J, Sandoval E, Cordero-Ampuero J, Molina-Manso D, Ortiz-Pérez A Fernández-Roblas $\mathrm{R}$, et al. Sonication of Intramedullary nails: clinically-related infection and contamination. Open Orthop J. 2012;6:255-60.

31. Bereza PL, Ekiel A, Auguściak-Duma A, Aptekorz M, Wilk I, Kusz DJ, et al. Identification of silent prosthetic joint infection: preliminary report of a prospective controlled study. Int Orthop. 2013;37(10):2037-43. doi:10.1007/ s00264-013-1955-9.

\section{Submit your next manuscript to BioMed Central and we will help you at every step:}

- We accept pre-submission inquiries

- Our selector tool helps you to find the most relevant journal

- We provide round the clock customer support

- Convenient online submission

- Thorough peer review

- Inclusion in PubMed and all major indexing services

- Maximum visibility for your research

Submit your manuscript at www.biomedcentral.com/submit
) Biomed Central 